Vol. 22, 2020/e14

\title{
Representaciones en Física: construcción y validación de un cuestionario para la Enseñanza Media Superior ${ }^{1}$
}

\section{Representations in Physics: Construction and Validation of a Questionnaire for Higher Secondary Education}

\author{
Fernando Flores-Camacho (1) https://orcid.org/0000-0001-6165-4946 \\ Leticia Gallegos-Cázares (1) https://orcid.org/0000-0002-1485-2867 \\ Cynthia Lima González (2) https://orcid.org/0000-0002-2188-4998 \\ (1) Universidad Nacional Autónoma de México \\ (2) Universidad de Texas \\ (Recibido: 30 de noviembre de 2016; Aceptado para su publicación: 2 de octubre de 2017)
}

Cómo citar: Flores-Camacho, F., Gallegos-Cázares, L. y Lima, C. (2020). Representaciones en Física: construcción y validación de un cuestionario para la Enseñanza Media Superior. Revista Electrónica de Investigación Educativa, 22, e14, 1-17. https://doi.org/10.24320/redie.2020.22.e14.1728

\section{Resumen}

Este trabajo da cuenta de la construcción de un cuestionario para determinar las representaciones de conceptos y procesos físicos en estudiantes de bachillerato. La elaboración del instrumento pasó por diferentes etapas de revisión y aplicación entre grupos de alumnos y expertos en Física. La versión final se aplicó a una muestra de 120 alumnos de 6o. año de bachillerato del Colegio de Ciencias y Humanidades de la UNAM. El instrumento transitó por diversos criterios de validez (pertinencia, inteligibilidad, completitud y estructura equivalente) y pruebas de confiabilidad (Alfa de Cronbach y Modelo de Crédito Parcial de Rasch), de tal manera que se obtuvo un instrumento confiable para caracterizar diversos tipos de representaciones externas, como son las respuestas escritas, los esquemas, las gráficas y los dibujos. Los resultados muestran que este tipo de cuestionarios puede contribuir a los nuevos enfoques en enseñanza de las ciencias centrados en las construcciones representacionales y en la dinámica de cambio del pensamiento de los estudiantes.

Palabras clave: Enseñanza de las ciencias, evaluación, representaciones.

\section{Abstract}

This paper report the construction of a questionnaire to investigate high school students' representations of physics concepts. The development of the instrument went through different stages of revisions and implementation among students and experts in Physics. The final version was applied to a sample of 120 high school students (Science and Humanities College, UNAM). Questionaire validity included item analysis of intelligibility, completeness, and structure equivalence. The Rasch Partial Credit Model analysis was conducted to identify the different levels of knowledge integration of students' responses. Alpha

\footnotetext{
1 Agradecemos la colaboración de Héctor Covarrubias Martínez, Manuel Cruz Cisneros, Fernando Vega Calderón, Felipe Cabrera Martínez y Reyna Alejandra Fonseca Velázquez en la realización de este proyecto.
} 
Cronbach was calculated (0.75) as a measure of reliability. Results show that the questionnaire characterizes properly the diverse types of external representations used by students -e.g. written answers, schemes, graphs and drawings. The results obtained through this kind of questionnaires can contribute to the new theoretical approaches in science education focused on representations and their dynamics of change in students' thinking.

Keywords: Science Education, assessment, representations.

\section{Introducción}

En las últimas tres décadas la investigación en el campo del aprendizaje de las ciencias ha transitado por varios enfoques y formas de enseñanza, y por sus correspondientes instrumentos de investigación y de evaluación. Por ejemplo, con el surgimiento del movimiento de las concepciones alternativas o ideas previas (Duit y Treagust, 1998; Wandersee et al., 1994) se desarrollaron diversos instrumentos para determinarlas en prácticamente todos los campos de las ciencias naturales. Para el caso de la Física, entre los instrumentos más utilizados se encuentran el Force Concept Inventory (FCI) de Hestenes et al. (1992), el Force and Motion Conceptual Evaluation (FMCE) de Thornton y Sokoloff (1998) y el Thermal and Transport Concept Inventory (TTCI) de Miller et al. (2011). Más reciente, se ha construido el Basic Mechanics Conceptual Understanding Test (bMcu) de Hofer et al. (2017), elaborado para estudiantes del nivel secundario, pues los instrumentos previos dan cuenta, principalmente, del nivel superior y medio superior.

Estos y otros desarrollos, como el de Caleon y Subramaniam (2010) para el caso de las ondas, han servido para analizar la comprensión de los conceptos científicos, las ideas previas y para evlauar procesos de cambio conceptual. Sin embargo, uno de los principales problemas que persiste es que, a pesar de los avances en la comprensión de las nociones científicas con procesos educativos orientados por las teorías de cambio conceptual, las ideas previas que los sujetos construyeron en algún momento de su desarrollo no se eliminan o "cambian" por otras, y aparecen, nuevamente, cuando el contexto lo facilita (Flores y Gallegos, 1999; Pozo, 2014). Así, ideas como asignar propiedades macroscópicas a entidades microscópicas (Flores-Camacho et al., 2007) aparecen en contextos externos a los escolares (clase, laboratorio, etc.).

Resultados como el descrito han llevado a replantear los procesos de construcción y aprendizaje de los conocimientos científicos y en varios de ellos se establece como condicionante el reconocimiento de la multi-representacionalidad (Gilbert, 2008; Kozma y Russell, 2005; Pozo, 2014), es decir, que los sujetos construyen representaciones diversas de procesos fenomenológicos en contextos específicos. De esta forma se ha ampliado el campo de análisis para el aprendizaje de las ciencias.

Este enfoque, centrado en las representaciones como elemento central del aprendizaje, abre un amplio panorama de cambio para los procesos educativos y hace necesario contar con instrumentos de indagación y evaluación adecuados.

Los distintos enfoques educativos requieren del desarrollo de instrumentos de evaluación e investigación que sean congruentes con sus supuestos y perspectivas. De la misma forma, una visión educativa centrada en las representaciones, el cambio representacional y la multi-representacionalidad requiere de instrumentos que no midan sólo los conocimientos o interpretaciones de conceptos -como los inventarios de ideas previas mencionados anteriormente- sino que puedan determinar las descripciones y el conjunto de elementos que componen las representaciones explícitas, sean éstas expresadas en un lenguaje gráfico, simbólico o en una combinación de ambos.

Un entorno representacional implica que el sujeto pueda explicitar no sólo un enunciado o relación con una situación, sino también qué lenguaje lo describe, qué relación guarda su experiencia previa sobre ese concepto y qué formas, además de las matemáticas, usa para describirlo, así como el entorno donde lo ubica y si su descripción cambia a medida que el estudiante avanza en su conocimiento de los temas científicos. 
Lo anterior justifica la relevancia de construir instrumentos de investigación y evaluación acordes con un proceso representacional y su dinámica. El presente trabajo describe la construcción de un instrumento para determinar las representaciones de los estudiantes del bachillerato que permita conocer las diversas formas descriptivas, interpretativas y explicativas de un tema como las fuerzas y el movimiento en colisiones. El documento presenta un breve marco sobre las representaciones y sus implicaciones en el aprendizaje de las ciencias, una descripción del proceso de estructuración del instrumento, la metodología seguida de validación, una muestra de las representaciones de los alumnos analizadas desde el marco propuesto y consideraciones finales sobre el uso y posibilidades del instrumento.

\subsection{Representaciones y aprendizaje de las ciencias}

Con el reconocimiento de las ideas previas (Duit y Treagust, 1998) se desarrollaron diversas teorías de cambio conceptual con diferentes aproximaciones epistemológicas y cognitivas (Flores, 2004), lo que contribuyó a mejorar los procesos didácticos y curriculares en la enseñanza de las ciencias (Duschl y Grandy, 2008). Sin embargo, esto no ha redituado en la comprensión de los conceptos científicos como se esperaba. Por ello, estas teorías se han replanteado para que se acerquen más a lo fenomenológico (elementos cognitivos con los que se interpretan procesos y concepciones), como son las representaciones y su dinámica de cambio, donde ciertos conceptos se relacionan con otros, así como con diversos elementos de la experiencia de los alumnos, formando un entramado complejo (Gilbert, 2008; Pozo, 2014).

La idea de representación implica que el sujeto genera una estructura con la que puede inferir propiedades o cualidades posibles de lo que representa. En esa estructura, y en sus posibilidades de hacer predicciones y generar explicaciones, radica la importancia de las representaciones, puesto que se manifiestan como elementos útiles para dar cuenta de procesos que satisfacen la coherencia mínima que los sujetos requieren para interpretar y funcionar en su entorno (Flores y Valdez, 2007).

La construcción de un conjunto representacional y de recursos cognitivos adecuados para el aprendizaje de la ciencia, apoya significativamente a los estudiantes para que desarrollen representaciones de mayor alcance explicativo. Es decir, que les permita hacer inferencias con diferentes formas de representación, como son la construcción de esquemas y modelos que hagan posible la comprensión fenomenológica desde distintas posibilidades interpretativas o, de manera equivalente, como plantea diSessa (2004), construir nuevas estrategias de lectura y clases coordinadas más amplias.

Las representaciones que pueden conocerse de los alumnos son las externalizadas o externas, es decir, son todos aquellos elementos icónicos o simbólicos con los cuales se denotan a los representados y que se enseñan a los alumnos por ser elementos de aprendizaje de la ciencia escolar. De tal forma que la gráfica del movimiento de un objeto es una representación externa, lo mismo que una ecuación, una oración, un esquema o una imagen; también lo son las representaciones dinámicas, como las interacciones y simulaciones o las secuencias temporales de imágenes. Junto a esas representaciones externas están todos los elementos culturales y materiales que las hacen posibles. El lenguaje, las matemáticas, las reglas de elaboración de las gráficas, etc. son, como apunta diSessa (2002), el pilar para el pensamiento que constituye lo externo al sujeto.

Las representaciones externas juegan, por tanto, un papel importante en el aprendizaje (Perkins, 1994). En especial, Prain y Tytler (2012) hacen notar que las representaciones externas contribuyen a la dimensión epistemológica, la cual involucra dos aspectos: los epistémicos propiamente dichos, donde la construcción de modelos y variables de las representaciones funcionan como elementos constrictores de los procesos de los sujetos, por ejemplo, el proceso de indagación y; los semánticos, donde se dota de significado a símbolos y herramientas simbólicas.

Los procesos de explicitación no son, en general, simples, por lo que requieren de diversos niveles de competencia o habilidades para expresarlos. Por ejemplo, Kozma y Russell (2005) describen cinco niveles de competencia de explicitación representacional: representación como elemento figural, nivel básico o primario de habilidad simbólica, uso sintáctico y semántico de representaciones formales, y uso reflexivo y retórico de las representaciones. Es por ello que un instrumento de investigación y/o evaluación que esté 
orientado hacia las posibilidades representacionales de los estudiantes implica que deben estar presentes en el mismo todos los niveles de explicitación.

\subsection{Construcción y desarrollo del instrumento}

La construcción de un instrumento que haga explícitas las representaciones de los sujetos requiere de la elaboración de cuestionamientos que den pie a diversos niveles de explicitación (Kozma y Russell, 2005), lo cual estaría en concordancia con lo que se ha denominado Conocimiento Integrado, es decir:

La habilidad para generar ideas científicamente relevantes y el uso de teorías, así como de evidencia empírica, para conectar ideas para la explicación de fenómenos científicos o bien, para justificar proposiciones acerca de un problema científico. El conocimiento integrado se puede caracterizar como un proceso cognitivo dinámico que puede ser capturado o reconocido en las explicaciones. (Lee et al., 2011, p. 116)

Lee et al. (2011) han mostrado que cuando se pretende determinar el Conocimiento Integrado de los alumnos se debe conocer la comprensión de los conceptos y su manejo ante situaciones diversas, sus formas de representación externa, así como el nivel de las explicaciones que logran estructurar. Los mejores instrumentos, desde este enfoque, son aquellos que tienen "ítems de explicación". Además, estos ítems deben permitir diversos niveles de explicación, esto es, que las respuestas y argumentos que generan puedan tener continuidad o mostrar situaciones de cambio. Atendiendo a estos aspectos de integración, continuidad y extensionalidad que deben propiciarse en el instrumento de investigación/evaluación, se proponen las siguientes características:

1. Presentar situaciones cotidianas fácilmente interpretables por los estudiantes. Esto implica que los sujetos puedan hacer uso de un marco representacional y de las formas usuales en las que describen esas situaciones.

2. Atender a conocimientos que, en principio, han sido analizados a lo largo de las trayectorias escolares de los alumnos. Esta característica, al considerar los elementos de conocimiento en los que los sujetos han tenido alguna experiencia escolar y extraescolar, va en acuerdo con el enfoque de Conocimiento Integrado, ya que no pretende proponer situaciones nuevas que exijan la construcción de hipótesis que no puedan justificar de manera amplia.

3. Posibilitar un proceso de reelaboración de explicaciones a lo largo del instrumento. Esta característica implica proporcionar a los sujetos diversos momentos de reelaboración de sus representaciones, lo que -además de estar de acuerdo con el Conocimiento Integrado- los conduce a construir representaciones de mayor complejidad.

4. Susceptibles de ser representados de diversas formas: verbal, por medio de explicaciones y descripciones; simbólica, por medio de símbolos matemáticos, ecuaciones u otros símbolos; gráfica, por medio de representaciones esquemáticas y de procesos.

Estas características abarcan los elementos necesarios para que, a partir de las respuestas de los sujetos, puedan determinarse patrones de respuesta que den indicios sobre los posibles marcos representacionales con que cuentan y de las disimilitudes entre estudiantes, ya que proceden de situaciones escolares diferentes -en lo referente al uso de diversas formas de representación externa en la escuela, por ejemplo el uso de simuladores, mapas conceptuales, gráficas, etc.

Temática de análisis. Si bien toda temática de la ciencia escolar es factible de analizarse, dadas las características y el propósito de este instrumento es necesario considerar un tema que presente, para su comprensión, un aporte de diversas formas de representación. Pero, además, que sea un tema investigado previamente para tener referentes comparables y que presente cierto nivel de dificultad de comprensión para que el instrumento pueda captar formas de argumentación y descripción y, por último, que forme parte del currículo de la asignatura de Física que cursan los alumnos. Dentro de esa caracterización, el tema 
de colisiones implica la comprensión de los conceptos de fuerza, aceleración, velocidad y, particularmente la segunda y Tercera ley de Newton, por lo que cumple con los requisitos planteados. Este tema es especialmente complejo puesto que, por ejemplo, se ha identificado que los estudiantes suelen asociar fuerza con movimiento, de tal modo que se interpreta la fuerza como propiedad del objeto, que las fuerzas de acción y reacción ocurren en un mismo objeto, entre otras muchas ideas previas sobre las fuerzas y el movimiento (Gómez y Pozo, 2009; Hestenes et al., 1992; Hierrezuelo y Montero, 1988;). Por lo que respecta al currículo, es un tema cuyos conceptos han sido motivo de los cursos desde la secundaria y ampliados en el bachillerato.

Estructura de los ítems. Como se ha apuntado, los ítems tienen una estructura orientada a la explicación. Sin embargo, en su construcción se siguieron las características definidas por Haladyna et al. (2002) y Lima (2009), que si bien presentan recomendaciones para la construcción de ítems de elección múltiple, varios de los criterios que proponen pueden ser adaptados para preguntas de integración. De entre los principales elementos considerados se tomaron en cuenta: Evitar ítems con truco; Utilizar un vocabulario simple; Poner la idea central en el texto; No usar oraciones en sentido negativo; Escribir preguntas en un contexto cotidiano.

Además de las consideraciones descritas, los ítems deben favorecer que los estudiantes expresen diversas formas de representación externa; por ello son secuenciados a partir de una problemática que encabeza el cuestionario. A partir de eso, cada ítem implica una demanda donde los sujetos requieren: a) describir en forma escrita explicaciones y descripciones de situaciones; b) elaborar esquemas y dibujos; c) hacer uso de los símbolos gráficos correspondientes al tema; d) construir e interpretar gráficas y e) hacer inferencias a partir de situaciones de medición posibles. Por otro lado, la secuencia de los ítems implica mayor nivel de profundidad en las explicaciones por lo que, en su conjunto, el instrumento debe poder dar cuenta del nivel de comprensión del tema científico que se aborda en el instrumento.

Descripción del Intrumento. El instrumento es un cuestionario que 22 preguntas divididas en dos situaciones. En la primera situación (12 preguntas: 1.1 a 1.12) se presenta una colisión entre vehículos (auto compacto y un tráiler) y sobre esa situación se desarrollan las primeras tres preguntas en torno a los daños y efectos que sufren los conductores y los vehículos en la colisión. Las siguientes tres preguntas plantean que los alumnos utilicen símbolos como vectores, construyan y expliquen gráficas del comportamiento de las fuerzas en la colisión. La pregunta 7 plantea situaciones posibles para medir las fuerzas durante la colisión, la pregunta 8 es sobre el efecto o no de la gravedad y la pregunta 9 hace referencia explícita a su comprensión de la Tercera ley de Newton, las restantes tres preguntas son semejantes a las tres primeras pero las condiciones de los autos que colisionan son diferentes (dos autos iguales).

En la segunda situación (10 preguntas: 2.1 a 2.10) se plantea el choque de una pelota con el piso. Las dos primeras preguntas son sobre los efectos del rebote con relación a la fuerza en el momento del impacto, la tercera pregunta es sobre su descripción gráfica y explicación del comportamiento de la fuerza en el momento del impacto y la siguiente pregunta sobre el comportamiento de la velocidad (pregunta 4). La pregunta 5 plantea la situación de la medición de la fuerza y la velocidad en el momento del choque, la pregunta 6 es sobre la acción de la gravedad y la pregunta 7 sobre la aplicabilidad de la Tercera ley de Newton y cómo la representarían. Las últimas tres preguntas plantean la misma situación con cambios en los materiales del suelo donde rebota la pelota (el cuestionario se presenta en el Anexo).

\section{Método}

La población a la que está destinado este instrumento son estudiantes del nivel bachillerato, quienes han tenido varios cursos de física desde la secundaria y en este nivel educativo cuentan, además, con actividades experimentales que de una u otra forma han contribuido a que tengan diversos elementos de representación de un conjunto básico de fenómenos y conceptos científicos. Adicionalmente, para una mejor caracterización, el instrumento se validó con estudiantes que han tenido cursos de ciencias en los nuevos laboratorios del bachillerato de la UNAM. Estos laboratorios presentan la posibilidad de que los alumnos exploren, además de la experimentación, con recursos digitales que posibilitan la toma de datos en tiempo real por medio de sensores y de las gráficas que se construyen en el transcurso de la medición, 
y de manera simultánea con el uso de simuladores, construcción de esquemas, toma de videos, consulta en Internet y trabajo colaborativo, lo que los convierte en espacios escolares idóneos para que los alumnos construyan, a través de múltiples representaciones externas, amplios marcos representacionales.

El proceso de validación del instrumento se aplicó con las siguientes muestras específicas: 30 alumnos de bachillerato en la fase de construcción del instrumento, 3 especialistas en Física (validación de expertos) y 120 estudiantes del bachillerato para la validación estadística. En las etapas de validación se describe la participación de cada muestra. Cabe destacar que las muestras fueron designadas por profesores voluntarios del Colegio de Ciencias y Humanidades (CCH) de la UNAM que aceptaron aplicar a sus alumnos el instrumento en sus distintas fases de construcción.

El proceso de validación del instrumento con fines de investigación tiene dos componentes: la validez del instrumento y la confiabilidad del mismo, criterios necesarios para garantizar que el instrumento mide lo que se espera y que no depende de situaciones específicas de aplicación. A continuación se describe el proceso seguido para cada uno de ambos componentes.

Para la validez del instrumento se siguieron los siguientes criterios:

Inteligibilidad de los ítems. Para determinar si las preguntas pueden ser comprendidas se llevó a cabo una prueba con 15 estudiantes de diversos semestres (primera versión). Del resultado, se modificaron aquellos ítems que inducían a los alumnos a respuestas alejadas de lo esperado.

Completitud de los ítems. Para garantizar que los ítems puedan ser respondidos de manera adecuada y que la información que proporcionan es suficiente para dar una respuesta amplia y correcta desde el punto de vista de la física, cada cuestionario se aplicó a tres profesionales (segunda versión del instrumento). Del análisis de sus respuestas se realizaron ajustes al Cuestionario, además de constituir la base para la construcción de la rúbrica que utilizó para la asignación de valores, como se describirá en la sección de confiabilidad.

Pertinencia de los ítems. Para este rubro se llevó a cabo la aplicación del cuestionario a estudiantes del semestre al que está destinado. Se aplicó a 15 estudiantes que cursaban la materia de Física en el cCH (tercera versión). La aplicación a los 15 estudiantes del nivel mostró que los ítems eran comprendidos de manera suficiente, y que proporcionan el desarrollo de ideas de forma clara en los alumnos para ser analizados en los aspectos esperados, la múltiple representacionalidad y el grado de comprensión de los conceptos físicos. Esta aplicación permitió conocer cómo responden los alumnos en función de las respuestas esperadas y hacer los últimos ajustes a los ítems para establecer la versión final. Con lo anterior se consideraron cubiertos los aspectos principales para garantizar la validez del cuestionario de acuerdo a Neumann et al. (2011).

Para obtener los valores y aplicar el criterio estadístico de confiabilidad se construyó una rúbrica para asignar valores a los ítems. El proceso de valoración de cada ítem con la rúbrica fue realizado por tres especialistas en Física y Didáctica de la ciencia, para garantizar criterios uniformes. A los datos se aplicó como criterio general de confiabilidad la consistencia interna (AERA et al., 2004) medida con el Alfa de Cronbach, y para analizar el comportamiento de los ítems y el equilibrio del instrumento se utilizó el Modelo de Crédito Parcial de Rasch (Lee et al., 2011; Yen y Fitzpatrick, 2006). La rúbrica utilizada se basa en lo propuesto por Wilson (2005), cuyos criterios se muestran en la tabla I. 
Tabla I. Criterios para la elaboración de Rúbricas

\begin{tabular}{ll}
\hline $\begin{array}{l}\text { Niveles de Integración } \\
\text { de conocimiento }\end{array}$ & Nivel y características de las respuesta a los ítems \\
\hline Vínculos complejos & $\begin{array}{l}\text { Nivel } 5 \text { Explicita tres o más conceptos e ideas relevantes } \\
\text { y elabora dos o más vínculos válidos entre ellas. } \\
\text { Nivel } 4 \text { Explicita al menos dos conceptos e ideas relevantes } \\
\text { y elabora un vínculo válido entre dos ideas. }\end{array}$ \\
Vínculos parciales & $\begin{array}{l}\text { Nivel } 3 \text { Explicita ideas o conceptos relevantes pero no elabora } \\
\text { adecuadamente vínculos entre ellos. }\end{array}$ \\
No hay Vínculos & Nivel 2 Explicita ideas no precisas y relacionadas. \\
Irrelevante & Nivel 1 Contiene ideas irrelevantes al contexto científico. \\
\hline No hay información & Nivel 0 No hay respuesta al ítem. \\
\hline Fuente: Adaptado de Wilson (2005).
\end{tabular}

\section{Resultados}

Para dar cuenta de la forma de aplicación de la rúbrica, en la tabla ll se muestra un ejemplo de su aplicación de acuerdo con los criterios descritos.

Tabla II. Ejemplo de aplicación de la rúbrica para asignación de valores para la pregunta 1.4*

\begin{tabular}{|c|c|c|c|c|c|}
\hline \multicolumn{6}{|c|}{ Niveles de logro esperados } \\
\hline Nivel 0 & Nivel 1 & Nivel 2 & Nivel 3 & Nivel 4 & Nivel 5 \\
\hline $\begin{array}{l}\text { No hay } \\
\text { información. }\end{array}$ & $\begin{array}{l}\text { Describe y } \\
\text { representa } \\
\text { conceptos que } \\
\text { no se } \\
\text { relacionan con } \\
\text { la pregunta. }\end{array}$ & $\begin{array}{l}\text { Describe y } \\
\text { representa uno } \\
\text { o dos } \\
\text { elementos del } \\
\text { nivel } 5 \text { pero no } \\
\text { hay coherencia } \\
\text { en las } \\
\text { respuestas. }\end{array}$ & $\begin{array}{l}\text { Describe y } \\
\text { representa al } \\
\text { menos } 3 \text { de los } \\
\text { elementos del } \\
\text { nivel 5, pero no } \\
\text { establece } \\
\text { relaciones } \\
\text { entre ellos. }\end{array}$ & $\begin{array}{l}\text { Describe y } \\
\text { representa al } \\
\text { menos } 4 \text { de los } \\
\text { elementos del } \\
\text { nivel } 5 \text { y hace al } \\
\text { menos una } \\
\text { relación } \\
\text { correcta entre } \\
\text { ellos o } \\
\text { descripción } \\
\text { gráfica } \\
\text { correcta. }\end{array}$ & $\begin{array}{l}\text { Describe y } \\
\text { representa } \\
\text { correctamente: } \\
\text { 1. La fuerza sobre el } \\
\text { auto. } \\
\text { 2. La fuerza sobre el } \\
\text { tráiler. } \\
3 \text { La fuerza durante } \\
\text { la interacción de los } \\
\text { vehículos en el } \\
\text { tiempo (gráfica). } \\
\text { 4. La fuerza de } \\
\text { gravedad. } \\
\text { 5. La Tercera ley de } \\
\text { Newton. }\end{array}$ \\
\hline
\end{tabular}

Nota: *Pregunta 1.4 En la siguiente figura correspondiente a esta situación (colisión entre dos vehículos), dibuja la fuerza o las fuerzas presentes en el momento del choque entre ambos vehículos. También, explica qué representa lo que has dibujado.

Los datos de todas las respuestas fueron analizados con el método de consistencia interna (SPSS) y con el Modelo de Crédito Parcial de Rasch (R statistics); los resultados se presentan en la tabla III.

Tabla III. Resultados de pruebas estadísticas

\begin{tabular}{c|c|c|c}
\hline Confiabilidad & \multicolumn{3}{|c}{ Modelo de Crédito Parcial de Rasch } \\
\cline { 2 - 4 } (Alpha de Cronbach) & Porcentaje por Nivel & Calibración & Dificultad \\
\hline 0.75 & Nivel 1;9\% & \pm 1 logit & -0.78 a 0.53 \\
& Nivel 2;20\% & & \\
& Nivel 3; 26\% & & \\
& Nivel 4; 35\% & & \\
& Nivel 5; $10 \%$ & & \\
\hline
\end{tabular}

La confiabilidad del cuestionario es de 0.75 medido con el Alfa de Cronbach, tomando la comparación de mitades de la muestra (split-half). 
El Modelo de Crédito Parcial de Rasch permitió tomar en cuenta los niveles de Conocimiento Integrado utilizado para la rúbrica a partir de los índices de dificultad de los ítems y del análisis de las respuestas, y establecer con ello el balance del instrumento. De acuerdo con estos resultados, más del $61 \%$ de los estudiantes de la muestra contestó en los niveles 3 y 4 . Esto significa que un poco más de la mitad de los estudiantes tiene una comprensión de regular a buena y representan, en general, de manera simbólica las fuerzas. Sin embargo, no alcanzan el nivel esperado, sobre todo en las representaciones de las fuerzas en el tiempo.

El 29\% de los estudiantes tuvo respuestas poco coherentes que muestran una comprensión deficiente del tema y pocos recursos de representación (niveles 1 y 2). El nivel 5, que correspondería al de experto, fue alcanzado por el $10 \%$ de la muestra-como se esperaba dados los antecedentes de la comprensión que los estudiantes tienen del tema de Fuerzas y que han sido puestos de manifiesto en evaluaciones realizadas con instrumentos como FCl y en el reciente trabajo de Bani-Salameh (2017).

\section{Discusión y conclusiones}

El proceso de validez, los datos de consistencia del instrumento y los de distribución de complejidad de los ítems (de acuerdo al Modelo de Crédito Parcial de Rasch) dan cuenta de un instrumento confiable y equilibrado para ser aplicado en alumnos de bachillerato. Como se describe a continuación, a través del instrumento es posible evaluar el nivel de representación científica de los alumnos.

Consideramos que el instrumento es efectivo para ese propósito por las siguientes razones: en primer lugar, las respuestas a los ítems muestran diferentes representaciones externas, evidenciadas por el diverso número de conceptos, símbolos, gráficas y relaciones que expresan los estudiantes en sus argumentos. En sus respuestas, los estudiantes usan argumentos expresados en ítems previos, indicando que tienen la oportunidad de reflexionar sobre su propio conocimiento y elaborar, a partir de su pensamiento inicial, el conjunto base de ideas necesarias para explicar los distintos tipos de interacción. Por ejemplo, las explicaciones acerca del daño en cada vehículo después de la colisión expresan una relación, de proporcionalidad directa, entre el daño y el tamaño del vechículo (respuesta incorrecta).

Cuando se pregunta acerca del momento de la colisión, el estudiante reelabora su respuesta en torno a su explicación inicial y agrega los conceptos de masa y aceleración. Así, los estudiantes tienen la oportunidad de refinar sus respuestas iniciales en las que la primera variable considerada es el tamaño, reelaborando sus ideas hasta establecer relaciones entre la masa y la fuerza. La secuencia de ítems se muestra adecuada para promover esas oportunidades de refinamiento y reelaboración sobre el pensamiento, que implica la construcción representacional de toda la problemática planteada en el instrumento.

En segundo lugar, podemos notar cómo, a partir del instrumento, es posible encontrar diversos niveles que indican representaciones de menor o mayor complejidad en términos conceptuales. Estos niveles de representación se distinguen a partir de la correspondencia entre lo que los estudiantes elaboran (escrito, gráfico o simbólico) y lo pertinente a la ciencia escolar (también en lo escrito, gráfico y simbólico). En la tabla IV se muestra el caso de dos alumnos que presentan distintos niveles de pertinencia en sus representaciones. 
Tabla IV. Descripción de la comparación entre las representaciones gráficas del comportamiento de la fuerza en el tiempo y la Tercera ley de Newton

\begin{tabular}{|c|c|c|}
\hline Concepto representado & Nivel 2 & Nivel 4 \\
\hline \multirow[t]{3}{*}{$\begin{array}{l}\text { Fuerza: Comportamiento en } \\
\text { el tiempo durante la colisión }\end{array}$} & fleds & frem \\
\hline & $\rightarrow$ linew (f) & 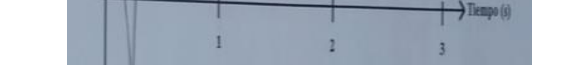 \\
\hline & $\begin{array}{l}\text { Su representación gráfica en el } \\
\text { momento del impacto no } \\
\text { corresponde al fenómeno físico. }\end{array}$ & $\begin{array}{l}\text { Su representación gráfica en el momento } \\
\text { del impacto corresponde al experimento } \\
\text { realizado en clase y al fenómeno físico. }\end{array}$ \\
\hline Tercera Ley de Newton & $\begin{array}{l}\text { Describe que la fuerza que } \\
\text { experimenta el tráiler es menor a la } \\
\text { que experimenta el automóvil }\end{array}$ & $\begin{array}{l}\text { Describe que la fuerza que sienten } \\
\text { ambos objetos durante el impacto tiene } \\
\text { la misma magnitud pero sentido } \\
\text { contrario. }\end{array}$ \\
\hline
\end{tabular}

Como puede notarse en la tabla IV, el alumno que tiene el nivel 2 dibuja una gráfica que no toma en cuenta que en el inicio de la colisión la fuerza es cero; por el contrario, su gráfica inicia con un valor que parece indicar que lo que está representando es lo que muchos alumnos describen con la idea previa de que el objeto "lleva una fuerza" que se termina cuando el objeto deja de moverse. La descripción que hace de la Tercera ley de Newton confirma lo anterior, pues es una especie de descripción del ímpetu y no de la fuerza. En cuanto a la representación gráfica del alumno que se ubica en el nivel 4, parece tener claro que la fuerza se da en el momento del choque, que crece y decrece rápidamente. También se observa una especie de oscilación que puede deberse a la realización de un experimento con sensores, que registra otro tipo de interacciones que pudeden ocurrir en el proceso. En este caso, la Tercera ley de Newton es enunciada de manera correcta.

El proceso y las características descritas para la elaboración del cuestionario muestran una posibilidad de construcción de instrumentos confiables para determinar y evaluar las representaciones de los sujetos en temas de Física, que proporcionen -además de los elementos de comprensión- las posibilidades interpretativas de los alumnos y, con ello, contar con mayores parámetros para conocer y analizar los procesos de construcción de nociones científicas en los alumnos que pueden ser útiles en el campo de la investigación conceptual y representacional. Por otro lado, el cuestionario aquí empleado tiene utilidad en el contexto escolar para evaluar la comprensión y niveles de conocimiento alcanzado en los temas específicos de los programas de Física.

El instrumento descrito permite conocer cómo los alumnos representan e interpretan los esquemas usuales de los libros de texto (por ejemplo, vectores y gráficas), así como la comprensión que alcanzan de los conceptos, pero sobre todo es útil para determinar cómo construyen -ante una situación física posible, la colisión de dos vehículos- un escenario o marco representacional, constituido a partir de elementos cotidianos y de la ciencia que se les presenta en la escuela, y sobre el cual elaboran posibles explicaciones e inferencias.

El instrumento y la metodología para elaborarlo que se presenta no es más que una propuesta que puede orientar el desarrollo de instrumentos equivalentes en otros temas de la Física, pero tiene el potencial de constituirse en un apoyo al reciente enfoque representacional en la investigación y enseñanza de las ciencias. Por otro lado, queda abierto el tema de cómo, con instrumentos como el descrito, se pueden llevar a cabo investigaciones sobre los niveles de complejidad representacional que contribuirían a una mejor comprensión de los procesos cognitivos y de conceptualización de los alumnos, tarea en la que deberá seguirse investigando. 


\section{Referencias}

American Educational Research Association, American Psychological Association y National Council on Measurement in Education. (2004). Standards for educational and psychological testing. AERA.

Bani-Salameh, H. N. (2017). How persistent are the misconceptions about force and motion held by college students? Physics Education, 52(1), 1-7.

Caleon, I. y Subramaniam, R. (2010). Development and application of a Three-Tier diagnostic test to assess secondary students' understanding of waves. International Journal of Science Education, 37(7), 939-961.

diSessa, A. (2004). Coordination and contextuality in conceptual change. En E. F. Redish y M. Vincentini (Eds.), Research on physics education: proceedings of the international school of physics "Enrico Fermi", (pp. 137-156). IOS Press.

diSessa, A. A. (2002). Why "conceptual ecology" es a good idea. En M. Limón y L. Mason (Eds.), Reconsidering conceptual change: issues in theory and practice (pp. 29-60). Kluwer Academic Publishers.

Duit, R. y Treagust, D. F. (1998). Learning in science- from behaviourism towards social constructivism and beyond. En B. Fraser y K. Tobin (Eds.), International handbook of science education, Part 1, (pp. 3-25). Kluwer.

Duschl, R. y Grandy, R. (2008). Reconsidering the character and role of inquiry in school science: framing the debates. En R. Duschl y R. Grandy (Eds.), Teaching scientific inquiry: recommendations for research and implementation (pp. 1-37). Sense Publishers.

Flores, F. (2004). El cambio conceptual: interpretaciones, transformaciones y perspectivas. Educación Química, 15(3), 256-269. http://dx.doi.org/10.22201/fq.18708404e.2004.3.66183

Flores, F. y Gallegos, L. (1999). Construcción de conceptos físicos en estudiantes. La influencia del contexto, Perfiles Educativos, 21(85), 90-103.

Flores, F. y Valdez, R. (2007). Enfoques epistemológicos y cambios representacionales y conceptuales. En J. Pozo y F. Flores (Eds.), Cambio conceptual y representacional en el aprendizaje y la enseñanza de la ciencia (pp. 21-35). Antonio Machado Libros.

Flores-Camacho, F., Gallegos-Cázares, L., Garritz, A. y García-Franco, A. (2007). Incommensurability and multiple models: representations of the structure of matter in undergraduate chemistry students. Science \& Education, 16, 775-800. https://doi.org/10.1007/s11191-006-9049-3

Gilbert, J. (2008). Visualization: an emergent field of practice and enquiry in science education. En J. K Gilbert, M. Reiner y M. Nakhleh (Eds.), Visualization: theory and practice in science education (pp. 1-2). Springer.

Gómez, M. y Pozo, J. (2009). Aprender y enseñar ciencia: del conocimiento cotidiano al conocimiento científico. Morata.

Haladyna, T., Downing, S. M y Rodríguez, M. C. (2002). A review of multiple-choice item-writing guidelines for classroom assessment. Applied Measurement in Education, 15(3), 309-333.

https://doi.org/10.1207/S15324818AME1503 5

Hestenes, D., Wells, M. y Swackhamer, G. (1992). Force concept inventory. The Physics Teacher, 30, 141-158. https://doi.org/10.1119/1.2343497

Hierrezuelo, M. J. y Montero, M. A. (1988). La ciencia de los alumnos. Laia/Ministerio de Educación y Ciencia. 
Hofer, S. I., Schumacher, R. y Rubin, H. (2017). The test of basic mechanics conceptual understanding (bmcu): using Rasch analysis to develop and evaluate an efficient multiple choice test on Newton's mechanics, International Journal of STEM Education, 4(18), 2-20. https://doi.ora/10.1186/s40594-017-0080-5

Kozma, R. y Russell, J. (2005). Students becoming chemists: developing representational competence, en J. Gilbert (Ed.), Visualization in science education (pp. 121-146). Springer.

Lee, H-S., Liu, O, L. y Linn, M. (2011). Validating measurement of knowledge integration in science using multiple-choice and explanation items. Applied Measurement in Education, 24(2), 115-136.

https://doi.org/10.1080/08957347.2011.554604

Lima, C. E. (2009). Changes in state of matter: a study of validity of Texas math and science diagnostic system (Tesis de maestría). Texas University.

Miller, R. L., Streveler, R., Yang, D. y Santiago Román A. I. (2011). Identifying and repairing student misconceptions in thermal and transport science: Concept inventories and schema training studies. Chemical Engineering Education, 45(3), 203-210.

https://ufdcimages.uflib.ufl.edu/AA/00/00/03/83/00193/AA00000383 00193.pdf

Neumann I., Neumann, K. y Nehm, R. (2011). Evaluating instrument quality in science education: Raschbased analyses of a nature of science test. International Journal of Science Education, 33(10), 1375-1405. https://doi.org/10.1080/09500693.2010.511297

Perkins, D. (1994). A new look in representations for mathematics and science learning. Instructional Science, 22, 1-37.

Pozo, J. I. (2014). Aprendizaje de la ciencia mediante múltiples sistemas de representación. En F. Flores (Ed.), Las tecnologías digitales en la enseñanza experimental de las ciencias: fundamentos cognitivos y procesos didácticos (pp. 13-31). unAM-Porrúa.

Prain, V. y Tytler, R. (2012). Learning through construction representation in science: a framework of representational construction affordances. International Journal of Science Education , 34(17), 2751-2773. https://doi.org/10.1080/09500693.2011.626462

Thornton, R. K y Sokoloff, D. R. (1998). Assessing student learning of Newton's laws: the force and motion conceptual evaluation and the evaluation of de active learning laboratory and lecture curricula, American Journal of Physics, 66(4), 338-352. https://doi.org/10.1119/1.18863

Wandersee, J., Mintzes, J. y Novak, J. (1994). Research on alternative conceptions in science. En D. Gabel (Ed.), Handbook of research on science teaching and learning (pp. 177-210). Mcmillan Publishing Company.

Wilson, M. (2005). Constructing measures: an item response modeling approach. Lawrence Erlbaum Associates.

Yen, W. M. y Fitzpatrick, A. R. (2006). Item response theory. En R. Brennan (Ed.), Educational Measurement (pp. 111-154). American Council of Education, Praeger. 


\section{Anexo}

CUESTIONARIO: Colisiones

Alumno(a):

Escuela:

Fecha:
Edad:

Semestre:

INSTRUCCIONES: Contesta las siguientes preguntas acerca de las dos situaciones presentadas.

\section{SITUACIÓN 1:}

En una autopista, un tráiler y un auto compacto chocan de frente. Afortunadamente, ambos conductores resultan ilesos...

1.1. ¿Cuál de estos dos vehículos sufre más daños? Explica el porqué.

1.2. ¿Cuál de los dos conductores habrá sentido más el choque: el del tráiler o el del auto compacto? Explica el porqué.

1.3. ¿El impacto del tráiler sobre el auto compacto es mayor, menor o igual que el impacto del auto sobre el tráiler? Explica el porqué.

1.4. En la siguiente figura correspondiente a esta situación, dibuja la fuerza o las fuerzas presentes en el momento del choque entre ambos vehículos. También, explica qué representa lo que has dibujado.

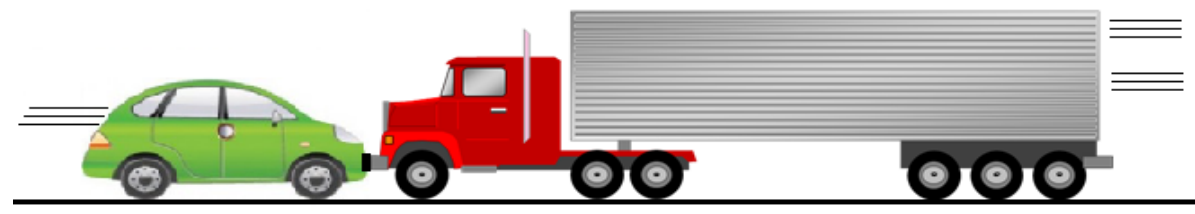


1.5. Suponiendo que el choque ocurrió durante tres segundos, desde el momento del impacto hasta que ambos vehículos quedaron en reposo, dibuja en la siguiente gráfica la fuerza (o las fuerzas) sobre el auto compacto. También, explica por qué se comporta así esa fuerza (o fuerzas) conforme a lo que dibujaste.

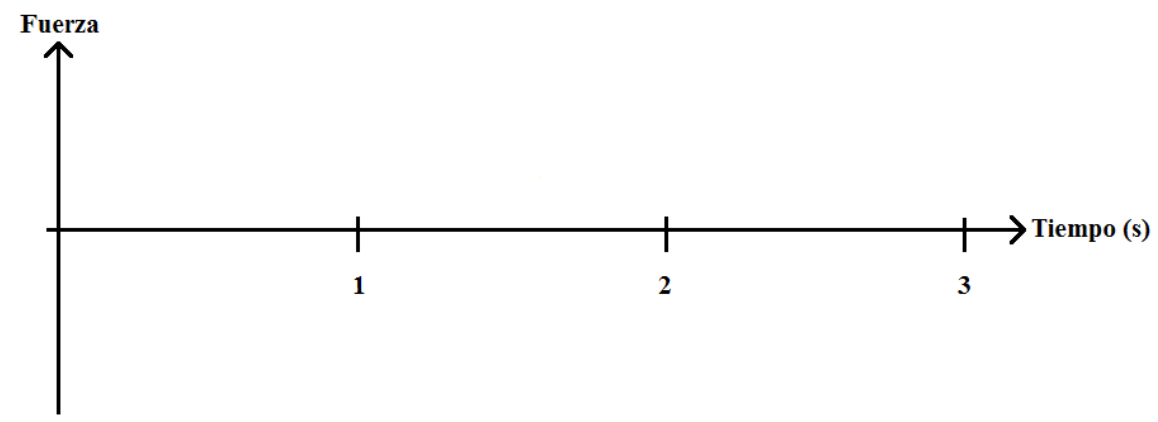

1.6. Ahora, en la siguiente gráfica dibuja la fuerza (o las fuerzas) sobre el tráiler durante ese mismo tiempo, y explica por qué se comporta así esa fuerza (o fuerzas) conforme a lo que dibujaste.

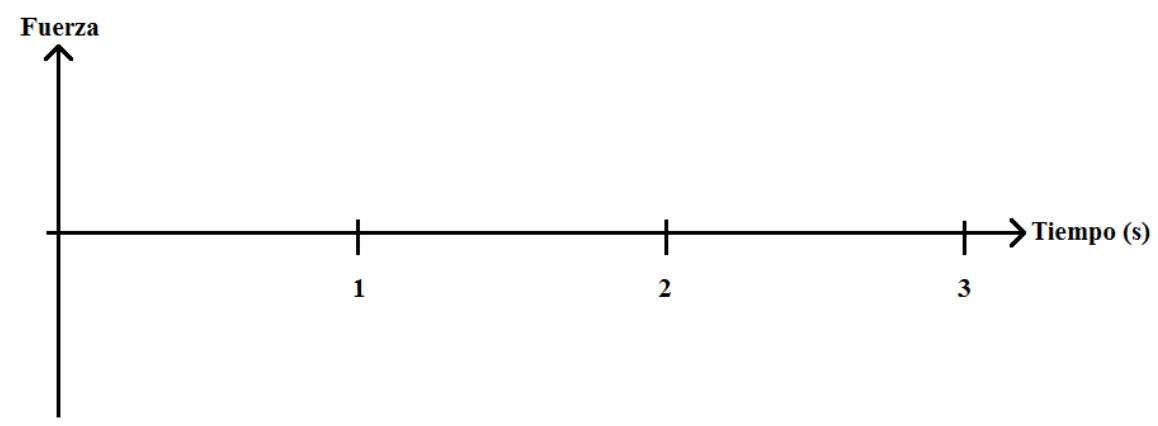

1.7. Tienes a disposición tres medios para registrar lo que ocurre durante el choque: a) un sensor de fuerza (registra el valor de la fuerza durante el choque en uno de los vehículos); b) un sensor de movimiento (registra el desplazamiento de uno de los vehículos); c) una cámara de alta velocidad (puedes registrar lo que ocurre durante el choque y después analizar cuadro por cuadro). Explica cómo te ayudarían estos dispositivos para obtener los valores de la fuerza que dibujaste en la pregunta 1.5 o en la 1.6. 
1.8. Explica cómo interviene la gravedad en esta situación.

1.9. Si alguien te preguntara si en esta situación vale o se aplica la Tercera ley de Newton, ¿qué le responderías y cómo le explicarías tu respuesta? Si lo requieres, para apoyar tu respuesta realiza uno o varios dibujos de tu explicación.

1.10. Si por ejemplo el choque fuera de frente entre dos autos compactos iguales, ¿cambiarían en algo esas fuerzas que consideraste anteriormente? Explica el porqué.

1.11. Ahora, ¿estos dos conductores de los autos compactos habrán sentido igual o diferente el choque? Explica el porqué.

1.12. En la siguiente figura, dibuja la fuerza o las fuerzas presentes en el momento del choque entre estos dos autos compactos iguales. También, explica qué representa lo que has dibujado.

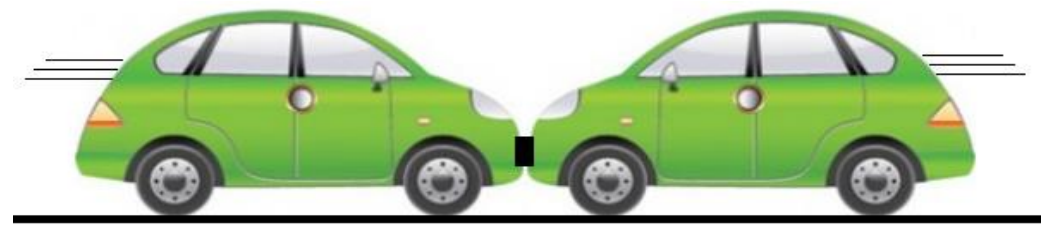




\section{SITUACIÓN 2:}

Manteniendo tu mano en posición horizontal sostienes una pelota. Después la sueltas, bota una vez en el suelo de concreto y sube; así como se muestra en la siguientes figuras:
A) La sueltas (baja):
B) Llega al suelo (bota):
C) Sube:
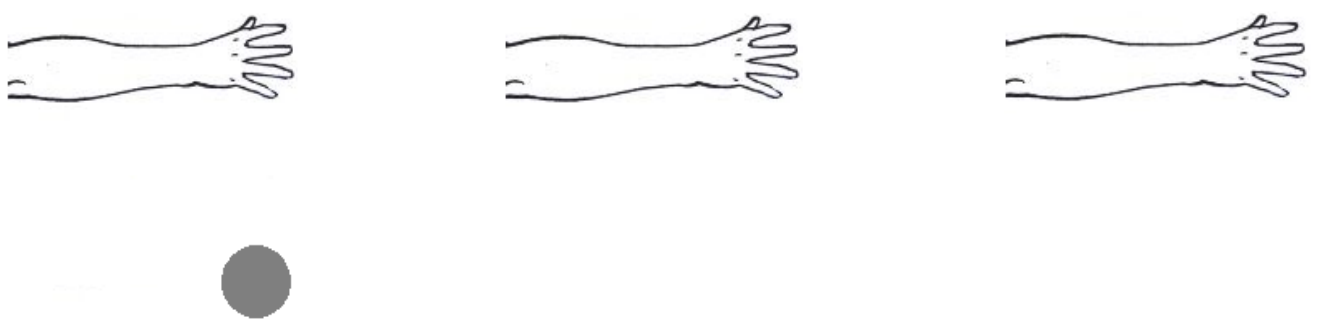

$=$

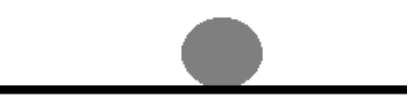

2.1. En las figuras anteriores, dibuja la fuerza o las fuerzas presentes cuando sueltas la pelota (A), cuando llega al suelo y bota (B) y cuando sube (C). También, explica qué representa lo que has dibujado.

2.2. Al momento en que la pelota llega al suelo y bota (B), ¿en cuál fue mayor la fuerza: en la pelota o en el suelo? Explica el porqué. 
2.3. En la siguiente gráfica, dibuja la fuerza (o las fuerzas) sobre la pelota en ese movimiento que hace al paso del tiempo. Explica por qué se comporta así esa fuerza (o fuerzas) conforme a lo que dibujaste.

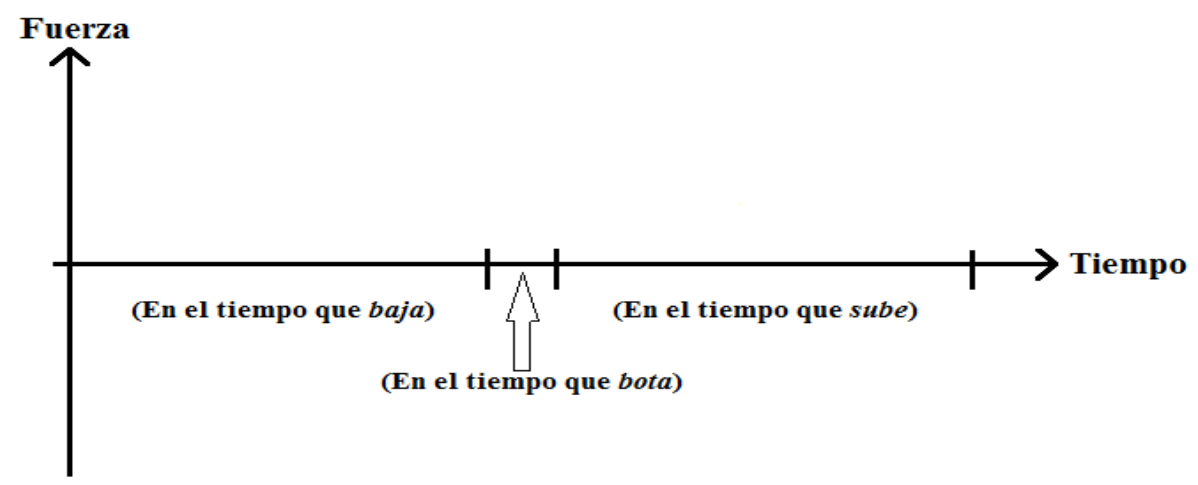

2.4. En la siguiente gráfica dibuja la velocidad de la pelota al paso del tiempo, y explica por qué se comporta así la velocidad conforme a lo que dibujaste.

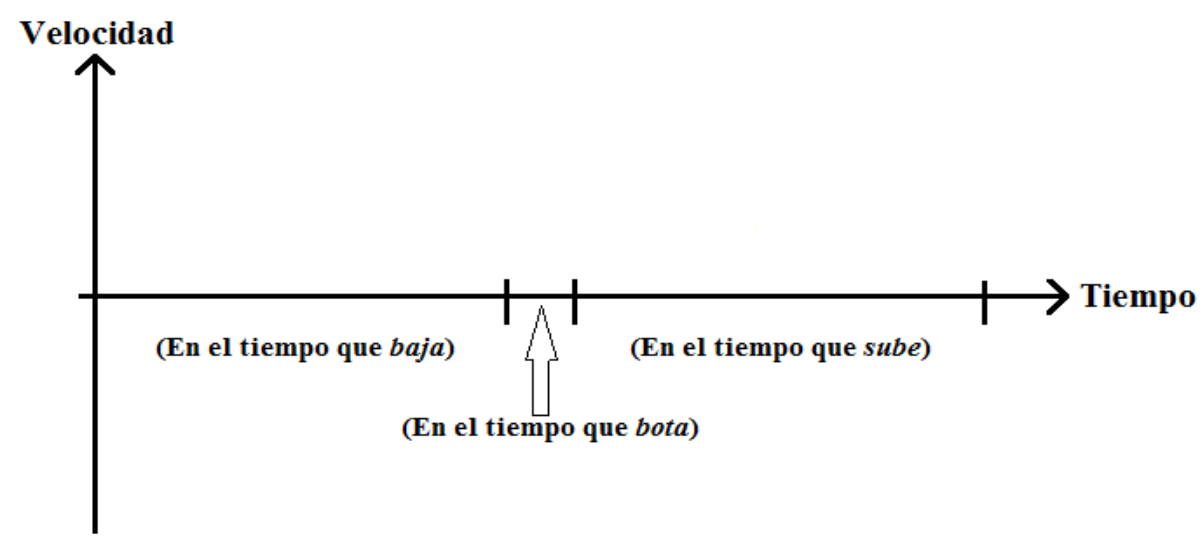

2.5. Tienes a disposición tres medios para registrar lo que ocurre durante el movimiento de la pelota: a) un sensor de fuerza (registra el valor de la fuerza durante la interacción con el piso); b) un sensor de movimiento (registra el desplazamiento de la pelota); c) una cámara de alta velocidad (puedes registrar lo que ocurre durante el movimiento y después analizar cuadro por cuadro). Explica cómo te ayudarían estos dispositivos para obtener los valores respectivos de la fuerza y de la velocidad, que dibujaste en

2.5.1. La gráfica de la fuerza o fuerzas (pregunta 2.3): 
2.5.2. La gráfica de la velocidad (pregunta 2.4):

2.6. Explica cómo interviene la gravedad en esta situación.

2.7. Si alguien te preguntara si en esta situación vale o se aplica la Tercera ley de Newton, ¿qué le responderías y cómo le explicarías tu respuesta? Si lo requieres, para apoyar tu respuesta realiza uno o varios dibujos de tu explicación.

2.8. Si por ejemplo el suelo fuera de un material suave, ¿cambiaría en algo el movimiento de la pelota a comparación de cuando el suelo era de concreto? Explica el porqué e indica en qué material suave piensas.

2.9. En este caso a comparación del anterior, ¿cambiaría en algo esa fuerza (o fuerzas) que consideraste en 2.1? Explica el porqué.

2.10. Ahora, al momento en que la pelota llega al suelo suave, ¿en cuál fue mayor la fuerza: en la pelota o en el suelo? Explica el porqué.

¡GRACIAS POR TU COLABORACIÓNi 